\title{
Quarkonium and Heavy Flavour Physics with ALICE at the LHC
}

\section{Gagliardi*}

INFN, Sezione di Torino

E-mail: Martino.Gagliardi@cern.ch

\section{for the ALICE Collaboration}

ALICE (A Large Ion Collider Experiment) aims at studying the behaviour of nuclear matter at high energy density and the transition to Quark Gluon Plasma (QGP), in ultra-relativistic heavy-ion collisions.

Quarkonia and open heavy flavour are regarded as sensitive probes of parton-medium interaction; their measurement is done in ALICE over a wide pseudo-rapidity range: $-0.9<\eta<0.9$ and $-4<\eta<-2.5$.

During the first year of LHC operation, ALICE has been taking p-p data at $\sqrt{s}=7 \mathrm{TeV}$ and $\mathrm{Pb}-\mathrm{Pb}$ data at $\sqrt{s_{N N}}=2.76 \mathrm{TeV}$.

Preliminary results on quarkonium and heavy flavour in p-p collisions are presented; the first heavy flavour signals observed in $\mathrm{Pb}-\mathrm{Pb}$ collisions are also shown.

Workshop on Discovery Physics at the LHC-Kruger 2010

December 05-10, 2010

Kruger National Park, Mpumalanga, South Africa

\footnotetext{
* Speaker.
} 


\section{Introduction}

The ALICE[1] experiment at the Large Hadron Collider[2] was designed to study nuclear matter in ultra-relativistic heavy-ion collisions, at energy densities one order of magnitude larger than that of ordinary nuclear matter. Under these conditions, finite temperature QCD calculations on the lattice ${ }^{1}$ predict a transition to a deconfined state of matter known as Quark-Gluon Plasma (QGP).

Heavy flavour particles are sensitive to the properties of the medium formed in heavy ion collisions. In particular: open charm and beauty mesons are sensitive to the energy density, through the mechanism of in-medium energy loss of heavy quarks; quarkonium production suppression by colour screening was one of the first proposed signatures for QGP formation[4]; charmonium regeneration due to the recombination of initially uncorrelated $c$ and $\bar{c}$ quarks may occur at LHC energies[5,6]. A detailed description of the physics motivations for heavy flavour measurements in heavy-ion collisions can be found in [7].

The measurement of open heavy flavour production in p-p collisions with ALICE has a twofold interest: as normalisation for the main observables in heavy-ion analysis and as a test of perturbative QCD and production models in kinematic regions where ALICE is complementary to the other LHC experiments.

\section{Experimental apparatus}

A complete description of the ALICE experiment can be found in [1]. A description of the detector configuration and running conditions in the 2010 p-p run has been provided separately in this conference[8].

Heavy flavour production measurement at mid-rapidity is done with the ALICE central detectors $(-0.9<\eta<0.9)$. The analyses presented here are based on the Inner Tracking System (ITS) for vertexing and tracking, the Time Projection Chamber (TPC) for tracking and PID and the Time of Flight detector (TOF) for PID. At forward rapidity, heavy flavour production is measured with the muon spectrometer $(-4<\eta<-2.5)$. The muon spectrometer is equipped with 5 tracking stations and a dedicated trigger system with programmable cut on the muon $\mathrm{p}_{T}$.

The mid-rapidity analysis is based on minimum-bias events; the forward rapidity analysis is based on muon triggered events. Luminosity normalisation is performed via a reference minimumbias cross section, measured in dedicated scans and known with an uncertainty of $10 \%$ when this presentation was made.

\section{Results on open heavy flavour}

\subsection{Heavy flavour at mid-rapidity via hadronic decays}

A number of heavy flavour hadronic decays are reconstructed in ALICE via invariant mass analysis. Here, we present results on the most advanced analyses: $\mathrm{D}^{0} \rightarrow \mathrm{K}^{-} \pi^{+}, \mathrm{D}^{+} \rightarrow \mathrm{K}^{-} \pi^{+} \pi^{+}$and $\mathrm{D}^{*+} \rightarrow \mathrm{D}^{0} \pi^{+}$. The crucial aspect for such channels is the spatial resolution for secondary vertexing: in the 2010 configuration, the SPD single hit resolution is $14 \mu \mathrm{m}$ [9]. The impact parameter

${ }^{1}$ See e.g. [3] 
resolution is $75 \mu \mathrm{m}$ at a momentum of $1 \mathrm{GeV} / \mathrm{c}$; it is $20 \mu \mathrm{m}$ at $20 \mathrm{GeV} / \mathrm{c}[10]$. Moreover, low- $\mathrm{p}_{T}$ tracking in the ITS is essential for the detection of the soft $\pi^{+}$in $\mathrm{D}^{*+}$ decays. Information from TPC and TOF allows for kaon identification at low $\mathrm{p}_{T}(<2 \mathrm{GeV} / \mathrm{c})$.

The measured charmed meson yields are corrected for acceptance and PID, selection and reconstruction efficiency. This is evaluated via detailed detector simulation. The total efficiency is similar for for $\mathrm{D}^{0} \rightarrow \mathrm{K}^{-} \pi^{+}$and $\mathrm{D}^{+} \rightarrow \mathrm{K}^{-} \pi^{+} \pi^{+}$: it turns out to be ranging from $1 \%$ at $\mathrm{p}_{T} \simeq 2 \mathrm{GeV} / \mathrm{c}$ to $10 \%$ at $\mathrm{p}_{T}>8 \mathrm{GeV} / \mathrm{c}$. Feed-down from B-mesons must also be properly taken into account: in this analysis, this is done via the FONLL[11] predictions for beauty.

The $\mathrm{p}_{T}$-differential cross sections for $\mathrm{D}^{0}$ and $\mathrm{D}^{+}$for $3 \mathrm{GeV} / \mathrm{c}<\mathrm{p}_{T}<12 \mathrm{GeV} / \mathrm{c}$, based on a sample of $10^{8}$ minimum-bias events, are shown in Fig. 1. The most important sources of systematic uncertainty are B feed-down subtraction $\left(\simeq 25 \%\right.$ at $\left.\mathrm{p}_{T} \simeq 10 \mathrm{GeV} / \mathrm{c}\right)$ and selection efficiency (10\%). pQCD predictions[11][12] are compatible with the measured cross sections.

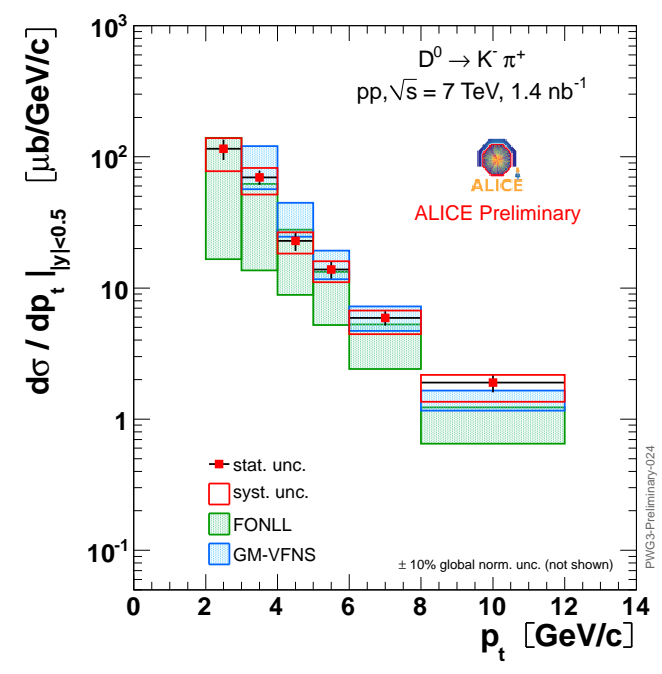

(a)

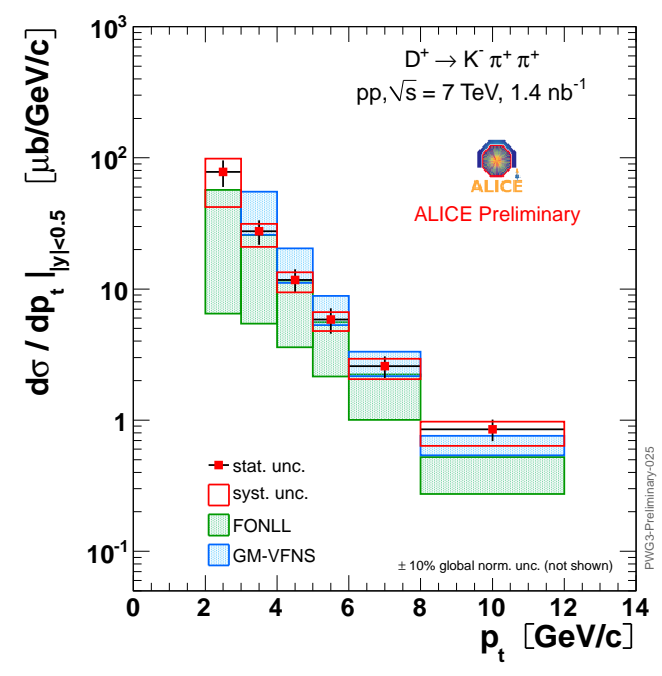

(b)

Figure 1: $\mathrm{p}_{T}$-differential $\mathrm{D}^{0}(\mathrm{a})$ and $\mathrm{D}^{+}(\mathrm{b})$ cross sections, based on a sample of $10^{8}$ minimum-bias events, as compared to the FONLL and GM-VFNS pQCD predictions.

The differential $\mathrm{p}_{T}$ spectrum $\mathrm{dN} / \mathrm{dp}_{T}$ for $\mathrm{D}^{*+}$ has also been measured and evaluation of the systematics is ongoing. The $\mathrm{D}^{0} / \mathrm{D}^{+}$and $\mathrm{D}^{0} / \mathrm{D}^{*+}$ ratios have been measured. The $\mathrm{p}_{T}$-integrated ratios were found to be $2.5 \pm 0.3$ and $2.2 \pm 0.3$, respectively, in agreement with measurements at lower energies by the ZEUS and $\mathrm{H} 1$ experiments (both reported in [13]) and by the CDF experiment[14].

The next analysis steps, where the full 2010 statistics will be used, include the extension of the $\mathrm{p}_{T}$ reach for $\mathrm{D}$ mesons and the estimation of B-feed down from data using vertex displacement. Analysis is also ongoing for several other channels for which signal has already been extracted, namely $\mathrm{D}^{0} \rightarrow \mathrm{K}^{-} \pi^{-} \pi^{+} \pi^{+}, \Lambda_{c} \rightarrow \mathrm{pK}^{-} \pi^{+}$and $\mathrm{D}_{s}^{+} \rightarrow \phi \pi^{+}$.

\subsection{Heavy flavour at mid-rapidity via semi-electronic decays}

Heavy flavour electrons are studied by measuring the inclusive single electron spectrum and subtracting a cocktail of the known non-heavy-flavour contributions. 
Electron identification is performed up to a few $\mathrm{GeV} / \mathrm{c}$ using the combined information of TPC and TOF. In order to minimise the effect of photon conversions, a hit in the first layer of the SPD (3.9 $\mathrm{cm}$ in radius) is required. Electron candidates are selected via a $3 \sigma$ time of flight $\mathrm{cut}^{2}$, which results in a clean rejection of low-momentum protons and kaons. Electron-pion separation is performed up to a momentum of $4 \mathrm{GeV} / \mathrm{c}$ via a cut on the specific energy loss, measured in the TPC with $\simeq 5 \%$ resolution. The residual contamination in the selected tracks is estimated via a double gaussian fit to the energy loss spectrum.

The electron sources implemented in the cocktail at this stage of the analysis are the Dalitz decays of $\pi^{0}, \eta, \eta^{\prime}, \rho, \omega, \phi$ and the conversions of secondary $\gamma$. The $\pi^{0}$ contribution is fixed using the measured ALICE $\pi^{0}$ spectrum[15], while the heavier mesons are implemented via $\mathrm{m}_{T}$ scaling. In further steps of the analysis, the contributions from direct photons and $\mathrm{J} / \psi$ will be added.

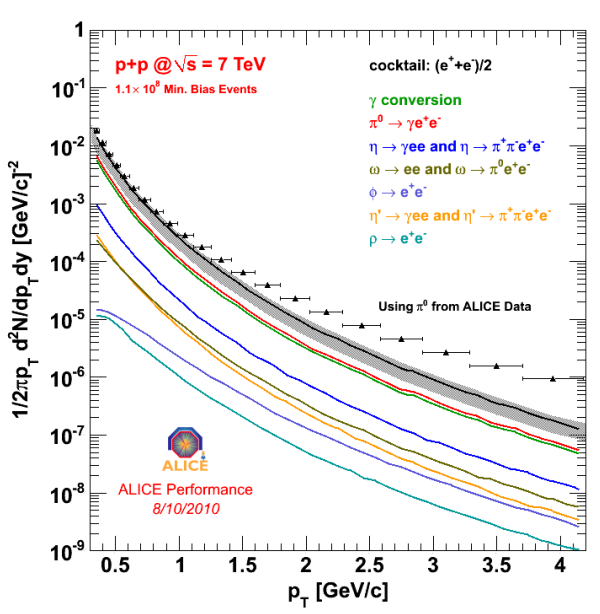

(a)

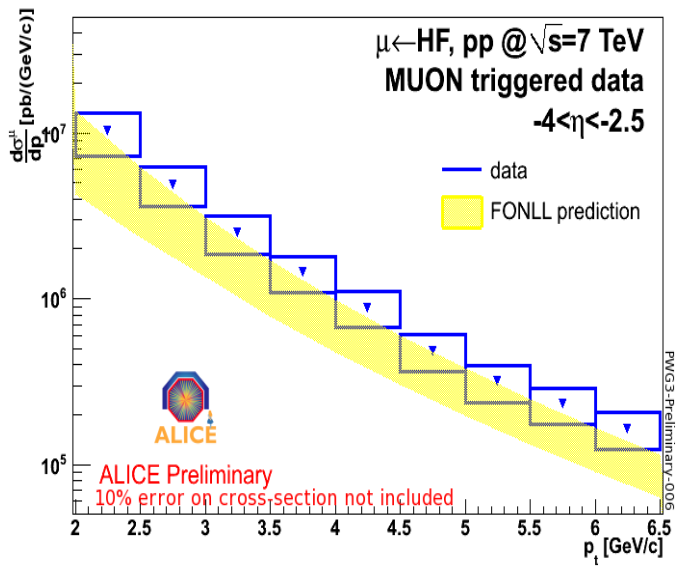

(b)

Figure 2: (a): $\mathrm{p}_{T}$ spectrum of single electrons obtained from a sample of $10^{8}$ minimum-bias events, compared to the electron cocktail; (b): $\mathrm{p}_{T}$ differential cross section for heavy flavour decay muons, compared to the FONLL calculation.

In Fig. 2(a) the measured electron spectrum (based on a sample of $10^{8}$ minimum-bias events), obtained after efficiency and acceptance correction, is shown and compared to the cocktail. An excess with respect to the cocktail, containing the heavy flavour signal, is clearly visible, increasing with $\mathrm{p}_{T}$.

Future steps in the analysis are: the luminosity normalisation, which will lead to the cross section for heavy flavour electrons; the separation of beauty-electrons with data-driven techniques (via secondary vertex displacement); the extension of the measurement to higher $\mathrm{p}_{T}$ using other detectors (Transition Radiation Detector and Electromagnetic Calorimeter).

\subsection{Heavy flavour at forward rapidity via semi-muonic decays}

Open heavy flavour production is studied in the ALICE muon spectrometer via the single muon spectrum. The momentum resolution of the spectrometer with the current alignment is about

\footnotetext{
${ }^{2}$ The total resolution on the time of flight measurement is about $130 \mathrm{ps}$.
} 
$12 \%$ at $\mathrm{p}_{T} \simeq 10 \mathrm{GeV} / \mathrm{c}$. In order to improve the rejection of hadronic background, matching in position is required between the tracks measured in the trigger and tracking systems ${ }^{3}$.

The main sources of background are "decay" muons (originating from decays of light mesons before the front absorber) and "secondary" muons (produced by light mesons in the front absorber). The analysis presented here is restricted to $\mathrm{p}_{T}>2 \mathrm{GeV} / \mathrm{c}$, where the secondary contribution is low $(\simeq 3 \%)$. The spectrum of decay muons is obtained by simulation. Its normalisation is fixed with low- $\mathrm{p}_{T}$ data. The background-subtracted spectrum is corrected for efficiency and normalised, to obtain the $\mathrm{p}_{T}$-differential cross section in $2 \mathrm{GeV} / \mathrm{c}<\mathrm{p}_{T}<6.5 \mathrm{GeV} / \mathrm{c}$ (Fig. 2(b)). The overall systematic uncertainty ranges from $20 \%$ to $30 \%$, decreasing with $\mathrm{p}_{T}$. The FONLL predictions for muons from charm and beauty decays [11] are compatible with our results.

The $\mathrm{p}_{T}$ reach of the spectrum will soon be extended via a refined alignment based on field-off data. Data-driven techniques for the subtraction of background are being studied.

\section{Results on quarkonia}

\section{1 $\mathrm{J} / \psi \rightarrow$ ee at mid-rapidity}

The preliminary results on (inclusive) $\mathrm{J} / \psi$ at mid-rapidity are based on a data sample corresponding to an integrated luminosity of $4 \mathrm{nb}^{-1}$. PID is performed via energy loss in the TPC; tracking uses both ITS and TPC. Cuts on the electron $\mathrm{p}_{T}(>1 \mathrm{GeV} / \mathrm{c})$ and pseudo-rapidity $(|\eta|<0.88)$ are performed. The signal is extracted by subtracting from the di-electron invariant mass spectrum the corresponding like-sign background distribution. The efficiency correction is performed via detailed simulation. The dependence of acceptance on the unknown $\mathrm{J} / \psi$ polarisation is a major source of systematic uncertainty. Here, we assume no polarisation and quote the polarisation-related uncertainty separately. The measured inclusive $\mathrm{J} / \psi$ cross section in $|\mathrm{y}|<0.88$ is:

$\left.\frac{d \sigma^{J / \psi}}{d y}\right|_{|y|<0.88}=7.36 \pm 1.22$ (stat. $) \pm 1.32$ (syst. $)_{-1.84}^{+0.88}($ syst.pol $) \mu b$. Besides polarisation, efficiency corrections are the main contribution to the systematic uncertainty $(\simeq 15 \%)$. The integrated cross section measurement is based on the best-calibrated subset of the data $(\simeq 100 \mathrm{~J} / \psi)$, corresponding to an integrated luminosity of $1.6 \mathrm{nb}^{-1}$. The whole data sample, for which luminosity normalisation is ongoing, has been used to measure the $J / \psi d N / d_{T}$, shown in Fig. 3(a).

\section{2 $\mathrm{J} / \psi \rightarrow \mu \mu$ at forward rapidity}

The preliminary results on (inclusive) $\mathrm{J} / \psi$ production at forward-rapidity in the muon spectrometer are based on a data sample corresponding to an integrated luminosity of $13.6 \mathrm{nb}^{-1}$. The event selection requires at least one reconstructed vertex in the SPD and position matching between the trigger and tracking information for at least one of the reconstructed muon tracks. A cut on the radial coordinate at the exit of the front absorber $\left(\mathrm{R}_{a b s}>17.5 \mathrm{~cm}\right)$ is also performed, in order to reject muons emitted at small angle, that have travelled a significant distance in the thick beam shield. The invariant mass spectrum is fitted with a Crystal Ball function and a double exponential for background. Trigger and tracking efficiency are evaluated via detailed simulation. As explained in Sec. 4.1, polarisation plays an important role in the systematic uncertainty. The measured inclusive cross section in $-4<y<-2.5$, based on a sample of $\simeq 1900 \mathrm{~J} / \psi$, is:

\footnotetext{
${ }^{3} \mathrm{An}$ iron wall with a total hadronic interaction length of $7 \lambda_{\text {int }}$ is located between the tracking and the trigger system.
} 


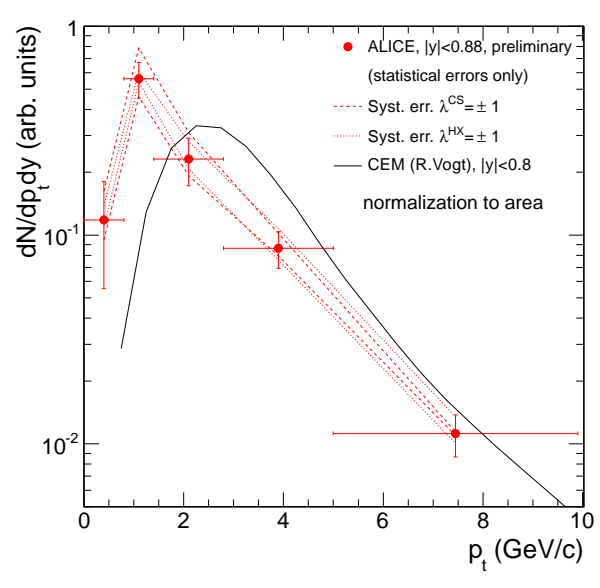

(a)

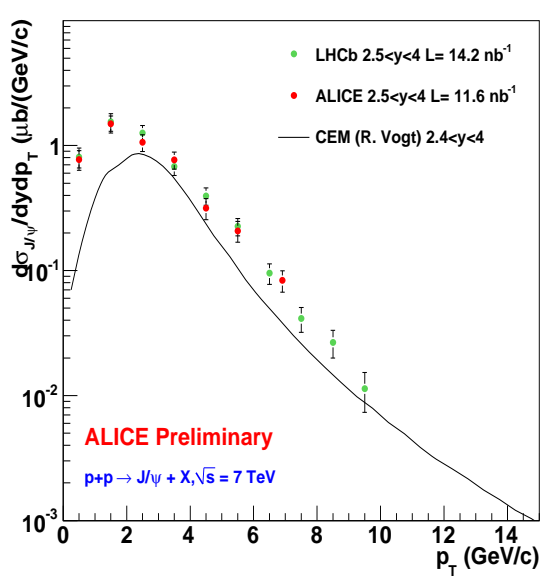

(b)

Figure 3: (a): inclusive $\mathrm{J} / \psi \mathrm{p}_{T}$ spectrum in $|\mathrm{y}|<0.88$; (b): inclusive $\mathrm{p}_{T}$-differential $\mathrm{J} / \psi$ cross section in $-4<y<-2.5$, as measured by ALICE and LHCb. The spectra in both figures are compared to an NLO Colour Evaporation Model calculation[16].

$\sigma_{-4<y<-2.5}^{J / \psi}=7.25 \pm 0.29$ (stat. $) \pm 0.98(\text { syst. })_{-1.27}^{+0.87}$ (syst.pol) $\mu b$. Such a result is in good agreement with the LHCb measurement[17] in the same rapidity window. The $\mathrm{p}_{T}$ and rapidity differential cross sections have also been measured (Fig. 3(b) and 4). In further steps of the analysis, the $\mathrm{p}_{T}$ reach will be extended by using the full available statistics.

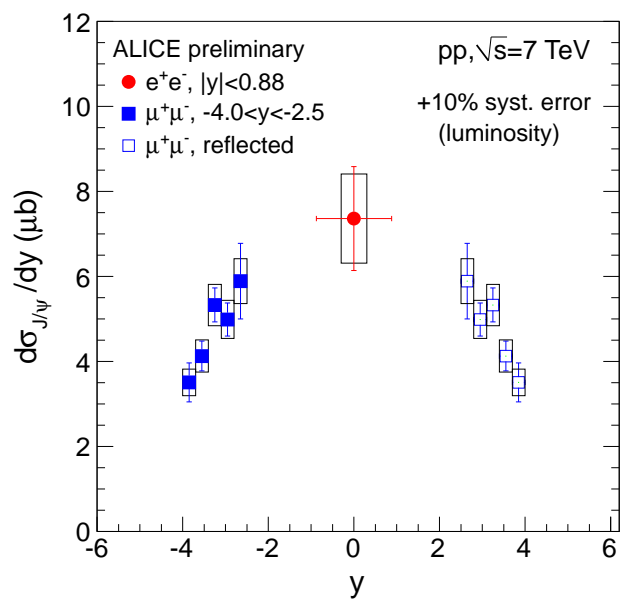

Figure 4: Inclusive rapidity-differential $\mathrm{J} / \psi$ cross section as measured by ALICE.

\section{Conclusions}

ALICE has provided preliminary results on open heavy flavour and quarkonium production in p-p collisions at $\sqrt{s}=7 \mathrm{TeV}$, at both mid- and forward rapidity. pQCD expectations are compatible with the ALICE preliminary results on open heavy flavour. The ALICE p-p results will provide a 
baseline for the interpretation of the $\mathrm{Pb}-\mathrm{Pb}$ data. The $\mathrm{Pb}-\mathrm{Pb}$ analysis is ongoing and the first heavy flavour signals in $\mathrm{Pb}-\mathrm{Pb}$ collisions have been observed (Fig. 5).
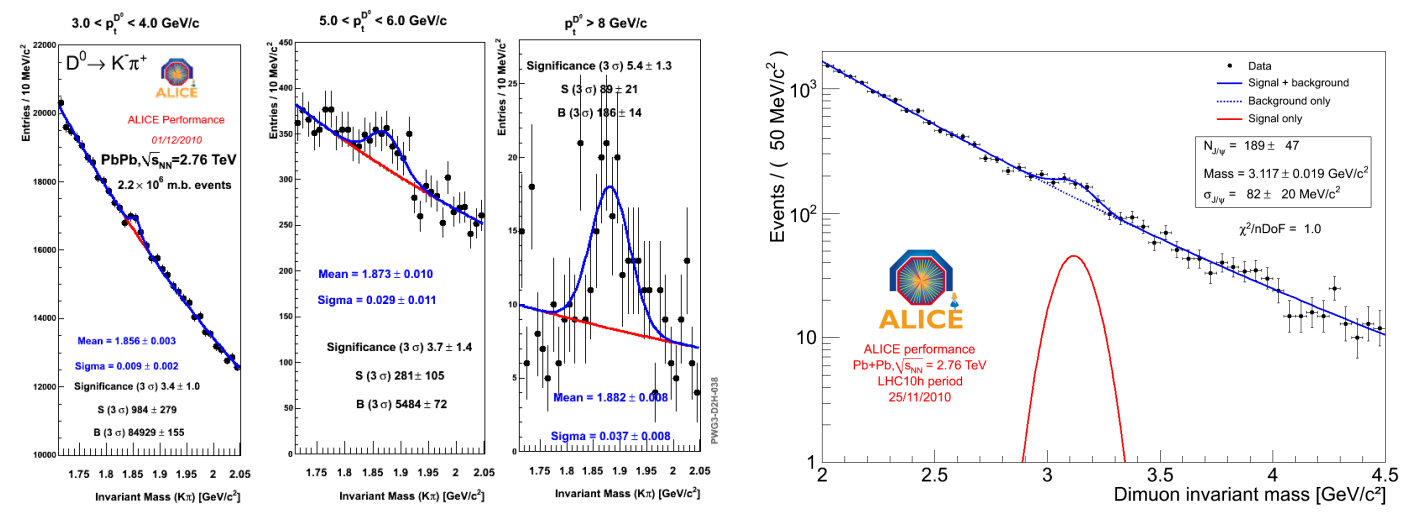

(a)

(b)

Figure 5: (a): $\mathrm{D}^{0} \rightarrow \mathrm{K}^{-} \pi^{+}$signal in $\mathrm{Pb}-\mathrm{Pb}$ collisions at $\sqrt{s_{N N}}=2.76 \mathrm{TeV}$, obtained from a sample of $2.2 \times 10^{6}$ minimum-bias events; (b): $\mathrm{J} / \psi \rightarrow \mu^{+} \mu^{-}$signal in $\mathrm{Pb}-\mathrm{Pb}$ collisions at $\sqrt{s_{N N}}=2.76 \mathrm{TeV}$, obtained from a sample of $2.6 \times 10^{6}$ minimum-bias events.

\section{References}

[1] K. Aamodt et al., JINST 3 (2008) S08002

[2] L. Evans et al., JINST 3 (2008) S08001

[3] F. Karsch, BNL-NT-06/2 [hep-lat/0601013]

[4] T. Matsui, H. Satz, Phys. Lett. B 178 (1986) 416

[5] P. Braun-Munziger, J. Stachel Phys. Lett. B 490 (2000) 196

[6] R.L. Thews et al., Phys. Rev. C 63 (2001) 054905

[7] B. Alessandro et al., J. Phys. G32 (2006) 1295

[8] P. Kuijer, in these proceedings

[9] K. Aamodt et al., JINST 5 (2010) P03003

[10] A. Dainese et al, proceedings of Hard Probes 2010 [hep-ex 1012.4036]

[11] M. Cacciari et al., private communication

[12] B. A. Kniehl et al., Phys. Rev. Lett. 96 (2006) 012001 and private communication

[13] S. Chekanov et al., JHEP 07 (2007) 074

[14] D. Acosta et al., Phys. Rev. Lett. 91 (2003) 241804

[15] K. Koch et al, proceedings of Hard Probes 2010 [hep-ex 1103.2217]

[16] R. Vogt, private communication

[17] G. Passaleva et al., PoS (ICHEP 2010) 213 